\title{
The Utility of the Artificial Taste Sensor in Evaluating the Bitterness of Drugs: Correlation with Responses of Human TASTE2 Receptors (hTAS2Rs)
}

\author{
Tamami Haraguchi, ${ }^{a}$ Takahiro Uchida, ${ }^{*},{ }^{a}$ Miyako Yoshida,${ }^{a}$ Honami Kojima, ${ }^{a}$ \\ Masaaki Habara, ${ }^{b}$ and Hidekazu Ikezaki ${ }^{b}$ \\ ${ }^{a}$ Faculty of Pharmaceutical Science, Mukogawa Women's University; 11-68 Koshien 9-Bancho, Nishinomiya, Hyogo \\ 663-8179, Japan: and ${ }^{b}$ Intelligent Sensor Technology Inc.; Atsugi, Kanagawa 243-0032, Japan. \\ Received August 1, 2017; accepted October 9, 2017
}

The purpose of this study was to examine the ability of the artificial taste sensor to evaluate the bitterness of drugs by comparing the responses of the taste sensor with documented responses of human TASTE2 receptors (hTAS2Rs). For this purpose 22 bitter compounds, used as ingredients of pharmaceutical medicines in Japan and known ligands of hTAS2Rs, were selected for testing. Their solutions $(0.01,0.03,0.1 \mathrm{~mm})$ were evaluated by five different taste sensors (AC0, ANO, BT0, C00, AE1). Correlations between physicochemical parameters of the compounds and the responses of the taste sensors and hTAS2Rs were evaluated. From taste sensor measurements, diphenidol, haloperidol, diphenhydramine, dextromethorphan and papaverine, all ligands of hTAS2R 10 and/or hTAS2R14, were predicted to express strong bitterness, surpassing that of quinine. Responses of taste sensors BT0 were found to be significantly correlated with responses of hTAS2R14. High $\log P$ values $(\geqq 2.73)$ and responses of hTAS2R14 were also significantly correlated $\left({ }^{* *} p<0.01\right.$, chi-square test). In conclusion, taste sensor BT0 is highly sensitive to bitterness and correlates significantly with hTAS2R14, making it useful for evaluating the bitterness of hydrophobic compounds which respond to hTAS2R14 and their inhibitors.

Key words taste sensor; human TASTE2 receptor; bitterness

It has been estimated that more than 700 compounds, including both plant-derived and synthetic compounds, such as amides, peptides, heterocyclic compounds, glycosides, alkaloids, terpenoids, phenols and flavonoids, are known to have a bitter taste. ${ }^{1)}$ The detection of bitterness is mediated by the TASTE2 Receptor (TAS2R) family of G protein-coupled receptors, and humans have been found to have 25 of these (hTAS2Rs). ${ }^{2-5)}$ Information on the association of many bitter ligands with particular TAS2Rs, established by in vitro assay, can be accessed via the Bitter DB database ${ }^{6)}$ (http://bitterdb. agri.huji.ac.il/dbbitter.php).

The taste of medicines is a critical factor in the development of orally administrated drug formulations, especially orally disintegrating tablets and liquids for older patients and children. ${ }^{7-9)}$ Huge efforts have been devoted by industry and academia in the development of taste-masked pharmaceuticals. $^{10,11)}$ However, compliance issues, stemming from the bitter taste of active pharmaceutical ingredients, are still common.

The standard method for evaluating taste, including the bitterness of drugs, is the human sensation test. However, this method has ethical and toxicological considerations and causes strain on subjects, while inter-subject variability has to be accounted for. Thus, the use of an artificial apparatus for taste evaluation has become popular. The taste sensor with lipid/polymer membranes developed by Toko is one such artificial chemical sensor, and is widely used in pharmaceutical applications. ${ }^{10-19)}$ A taste sensor must respond consistently to the same taste, like the human tongue. Bitter materials are adsorbed on the hydrophobic part of the membrane and cause a change in membrane potential by changing the charge density. Kobayashi et al. found that lipid/polymer membrane sensor selectivity for each taste is improved by modulating both the hydrophobic interaction between the taste sensor and bitter substance and the membrane charge density. ${ }^{12)}$ Correlation between results from the taste sensor and taste intensities recorded in human sensation testing has proven the usefulness of the taste sensor in predicting the taste of various substances. ${ }^{12-19)}$ However, there have been no reports examining the detailed correlation between the taste sensor response and that of human taste receptors.

The purpose of this study was to evaluate the correlation between the responses of five artificial bitter/astringency taste sensors composed of lipid/polymer membranes (AC0, AN0, BT0, C00 and AE1) produced by Intelligent Sensor Technology Inc. (Insent) and the responses of human TASTE2 receptors (hTAS2Rs), using 22 known bitter compounds, common ingredients of pharmaceutical medicines in Japan.

\section{Experimental}

Materials Twenty-two compounds: acetaminophen, azathioprine, caffeine, chloramphenicol, chlorpheniramine maleate salt, colchicine, cromolyn sodium, dextromethorphan hydrobromide monohydrate, diphenhydramine hydrochloride, diphenidol hydrochloride, erythromycin, famotidine, flufenamic acid, haloperidol, hydrocortisone, methimazole, noscapine, ofloxacin, papaverine hydrochloride, propylthiouracil, quinine hydrochloride and strychnine nitrate were purchased from Wako Pure Chemical Industries, Ltd., Osaka, Japan.

\section{Methods}

Taste Sensor Measurement

The taste sensor SA402B (Intelligent Sensor Technol- 


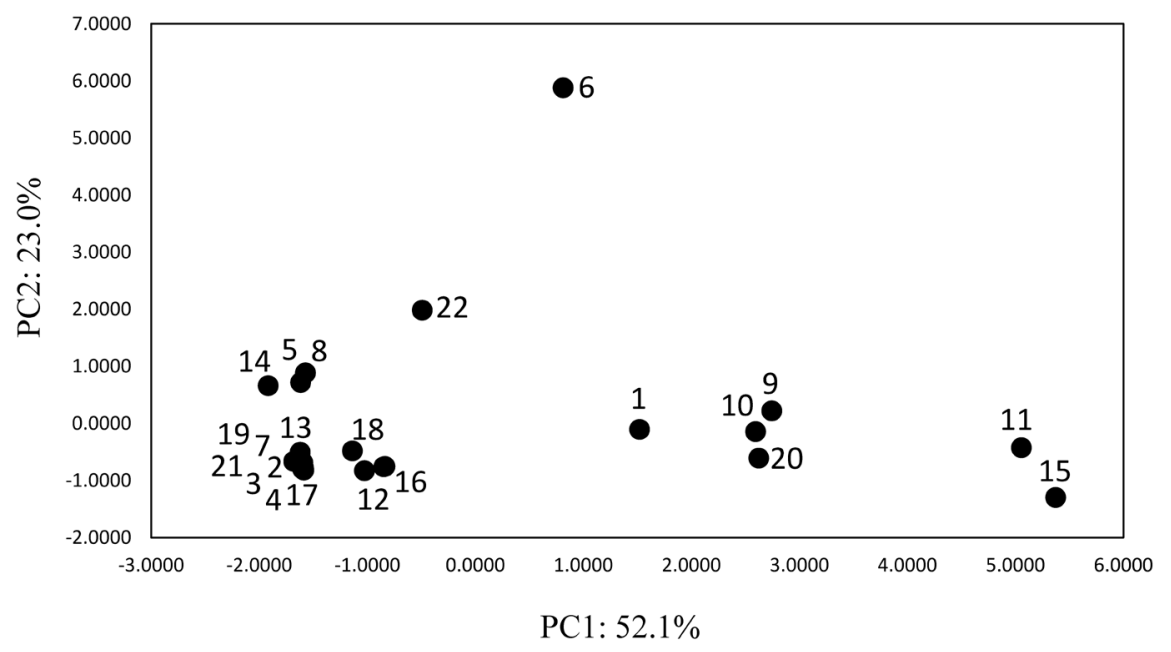

1 Quinine

2 Acetaminophen

3 Azathioprine

4 Caffeine

5 Chloramphenicol

6 Chlorpheniramine

7 Colchicine

8 Cromolyn

9 Dextromethorphan

10 Diphenhydramine

11 Diphenidol

12 Erythromycin

13 Famotidine

14 Flufenamic acid

15 Haloperidol

16 Hydrocortisone

17 Methimazole

18 Noscapine

19 Ofloxacin

20 Papaverine

21 Propylthiouracil

22 Strychnine

Fig. 1. Taste Map Obtained by Principal Component (PC) Analysis of Five Taste Sensor Outputs in Response to 22 Compound (0.1 mm)

ogy Inc., Atsugi, Japan) used in this study was the same as that used in our previous studies. ${ }^{14-17)}$ The detecting sensor part of the equipment consists of a reference electrode and a taste sensor which acts as the working electrode and is composed of various types of lipid/polymer membrane. ${ }^{12)}$ The lipid/polymer membranes used as bitter taste sensors in this study were as follows: AC0 (hexadecanoic acid/dioctyl phenylphosphonate), AE1 (tetradodecylammonium bromide/ dioctyl phenylphosphonate), AN0 (phosphoric acid di- $n$-decyl ester/dioctyl phenylphosphonate), BT0 (phosphoric acid di- $n$ decyl ester/bis(1-butylpentyl) adipate, tributyl $O$-acetylcitrate) and $\mathrm{C} 00$ (tetradodecylammonium bromide/2-nitrophenyl octyl ether). The 22 compounds tested (see above) were each dissolved in water to form solutions of $0.01,0.03$ and $0.1 \mathrm{~mm}$, prior to analysis by the taste sensors. The taste sensor outputs of the 22 compounds were analyzed by principal component analysis (PCA) using Ekuseru-Toukei 2010 (Social Survey Research Information Co., Ltd.).

Response Profiles of hTAS2Rs Stimulated with Drugs by in Vitro Assay

The response profiles of hTAS2Rs stimulated with the drugs used in this study were derived from the literature and the Bitter DB database. ${ }^{3,4,6)}$ Meyerhof et al. have systematically investigated the molecular receptive ranges of bitter taste receptors using natural or synthetic bitter chemicals to challenge all 25 human TAS2Rs in transfected cells. ${ }^{3)}$

Correlation between Taste Sensor and hTAS2Rs Responses

Taste sensor (AN0, AC0, BT0, C00, AE1) outputs for each of the 22 compounds were replaced by either 1 (response) or 0 (no response) as follows: If the taste sensor response increased in a dose-dependent manner and the response value at $0.1 \mathrm{~mm}$ was $\geq 2 \mathrm{mV}$, the response of the compound was designated as 1. If the taste sensor response did not increase in a dose-dependent manner and the response value at $0.1 \mathrm{~mm}$ was $<2 \mathrm{mV}$, the response of the compound was designated as 0. Correlations between taste sensor responses and hTAS2Rs responses cited from the literature were calculated and Fisher's exact tests were carried out using Ekuseru-Toukei 2010.

Correlation between Physicochemical Parameters and Responses of Taste Sensors and hTAS2Rs

Molecular weight $(\mathrm{MW}), \log P$ (log of the octanol-water partition coefficient using Ghose and Crippen's method) as a measure of hydrophobicity, $\left.{ }^{20}\right) \log S$ (log of the solubility in $\mathrm{mol} / \mathrm{L}$ ) as a measure of water solubility, ${ }^{21)}$ hydrogen bond donor (HBD) count, hydrogen bond acceptor (HBA) count, ring count in the smallest set of smallest rings and aromatic ring count extracted from the Bitter DB, were used as physicochemical parameters. These parameters were replaced by either 1 (high) or 0 (low) as follows: If the parameter of a compound was equal to or larger than that of quinine, the parameter was given the value 1 ; if the parameter was less than that of quinine, it was given the value 0 . Correlation coefficients between physicochemical parameters and responses of taste sensors and hTAS2Rs were calculated and Fisher's exact tests carried out using Ekuseru-Toukei 2010.

\section{Results}

Taste Sensor Measurements Quinine, diphenidol, diphenhydramine, dextromethorphan, papaverine, haloperidol, chlorpheniramine and noscapine responded to AC0. Quinine, diphenidol, diphenhydramine, dextromethorphan, papaverine, haloperidol and famotidine responded to AN0. Erythromycin, chlorpheniramine and strychnine responded slightly to AN0 but the value was $<2 \mathrm{mV}$ at $0.1 \mathrm{~mm}$. Erythromycin, ofloxacin, quinine, chlorpheniramine, diphenidol, dextromethorphan, noscapine, papaverine, haloperidol, diphenhydramine and strychnine responded to BT0. Azathioprine, acetaminophen, ofloxacin, fulfenamic acid, chloramphenicol and famotidine responded to $\mathrm{C} 00$. Cromolyn, chloramphenicol, chlorphenir- 
(a) Quinine
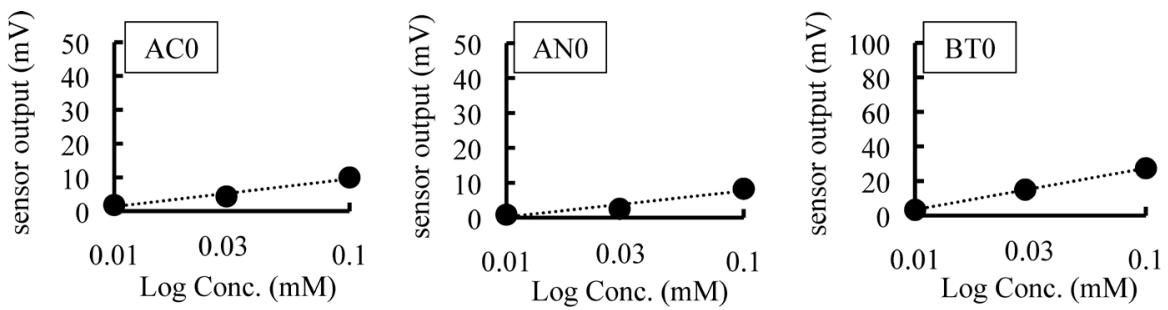

(b) Diphenidol
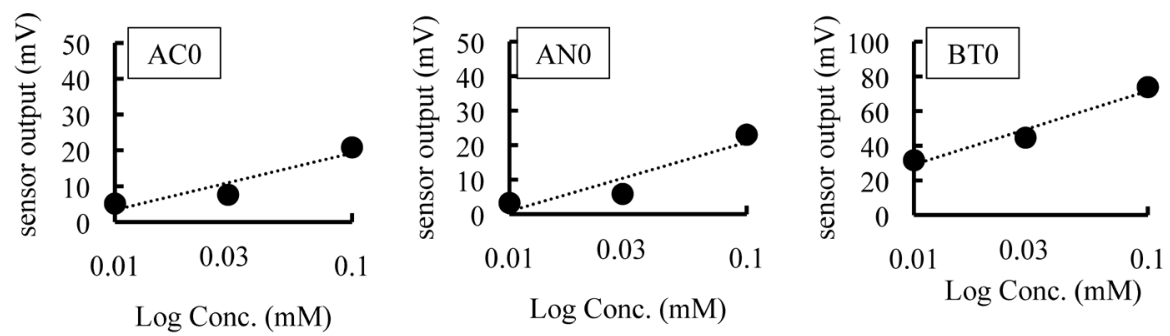

(c) Diphenhydramine
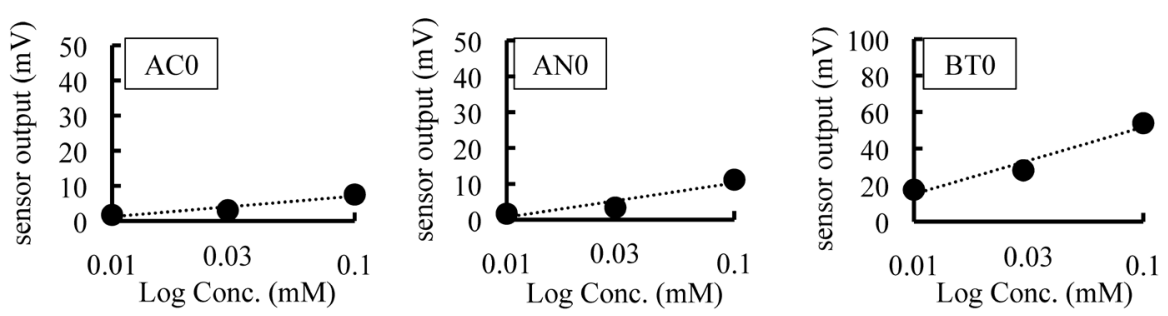

(d) Dextromethorphan
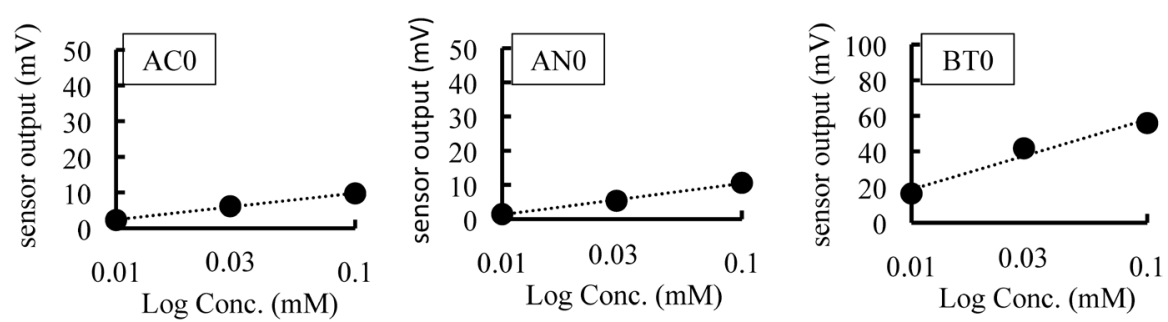

(e) Papaverine
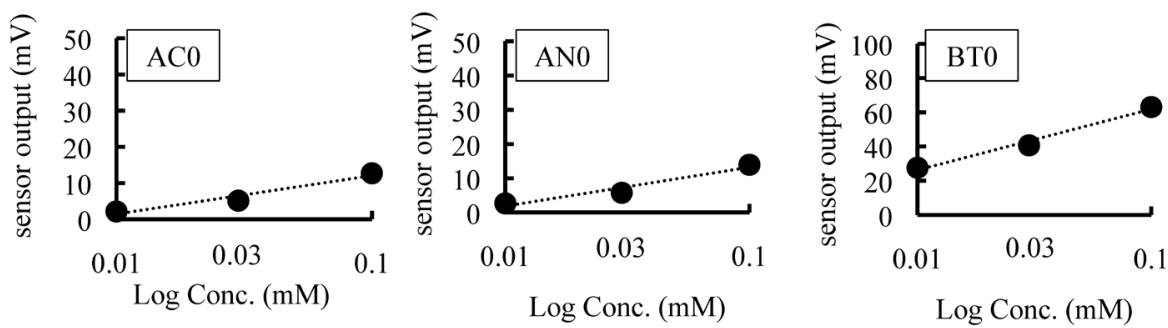

(f) Haloperidol
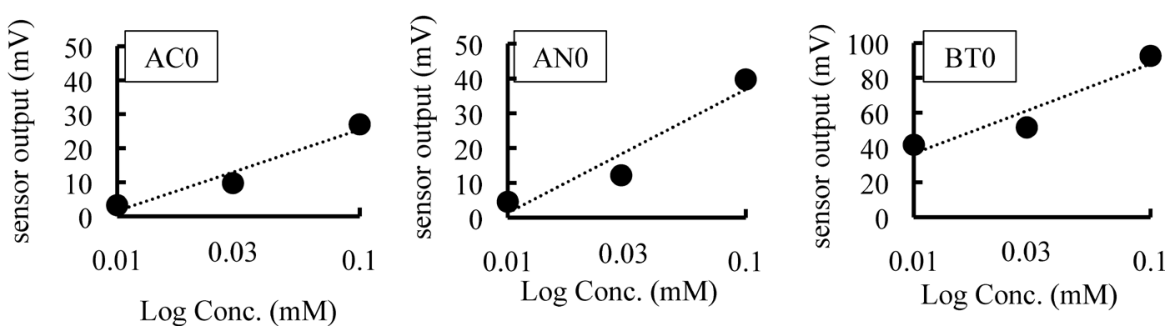

Fig. 2. Taste Sensor (AC0, AN0 BT0) Outputs in Response to Quinine (a), Diphenidol (b), Diphenhydramine (c), Dextromethorphan (d), Papaverine (e) and Haloperidol (f)

$n=3$, mean \pm S.D.

amine and strychnine responded to AE1. Caffeine, colchicine, hydrocortisone, propylthiouracil and methimazole did not respond to any of the five taste sensors.

The taste map obtained by PCA based on the five taste sen- sor outputs is shown in Fig. 1. PCA was used to estimate the largest and second-largest relative contributing factors (PC1 and $\mathrm{PC} 2$, respectively). The relative contributions of $\mathrm{PC} 1$ and PC2 were 52.1 and $23.0 \%$, respectively. Taste sensor outputs 
of $\mathrm{AN} 0, \mathrm{AC} 0$ and $\mathrm{BT} 0$ contributed to $\mathrm{PC1}$ rather than $\mathrm{PC} 2$, while those of $\mathrm{AE} 1$ and $\mathrm{C} 00$ contributed to $\mathrm{PC} 2$ rather than PC1. Diphenidol, haloperidol, diphenhydramine, dextromethorphan and papaverine were predicted to express strong bitterness, surpassing that of quinine, which was used as the standard of bitterness. Figure 2 shows the taste sensor (ACO, AN0, BT0) outputs of these six compounds. The BT0 taste sensor output values of these compounds were higher than those of AC0 or AN0.

Response Profiles of hTAS2Rs Stimulated with Drugs in in Vitro Assays The response profiles of hTAS2Rs stimulated with the 22 drugs used in this study were extracted from the Bitter DB. These are summarized in Table 1. Some compounds respond to a broad range of hTAS2Rs, whereas others respond to only one. Quinine and the five compounds (diphenidol, haloperidol, diphenhydramine, dextromethorphan, papaverine) predicted to express the strongest bitterness in this study, were shown to be ligands of hTAS2R10 and/or hTAS2R14.

Correlation between Taste Sensor and hTAS2Rs Responses Correlation coefficients between taste sensor responses and the responses of hTAS2Rs cited from the Bitter
DB are shown in Table 2. High correlation coefficients $(>0.7)$ were observed between the responses of AC0 and hTAS2R14 $(0.72)$ and slight correlation coefficients $(0.4<,<0.7)$ were observed between the responses of AC0 and hTAS2R40 (0.53), between AN0 and hTAS2R10 (0.43), hTAS2R14 (0.42) and hTAS2R44 (0.58), between BT0 and hTAS2R14 (0.46), and between AE1 and hTAS2R8 (0.46) and hTAS2R41 (0.46). Fisher's exact tests were carried out for these correlations.

Correlation between the responses of AC0 and hTAS2R14: AC0 responded to seven compounds (quinine, diphenidol, diphenhydramine, papaverine, haloperidol, chlorpheniramine and noscapine) out of the nine compounds known to be ligands of hTAS2R14; AC0 did not respond to 12 compounds (azathioprine, acetaminophen, erythromycin, ofloxacin, cromolyn, chloramphenicol, colchicine, strychnine, hydrocortisone, famotidine, propylthiouracil and methimazole) out of the 13 compounds which are not ligands of hTAS2R14. AC0 and hTAS2R14 responses were significantly correlated $(* * p<0.01$, Fisher's exact test).

Correlation between responses of AC0 and hTAS2R40: AC0 responded to three compounds (quinine, diphenidol and chlorpheniramine), all known to be ligands of hTAS2R40; AC0 did

Table 1. Response Profiles of hTAS2Rs Stimulated with 22 Compounds Cited from Bitter DB ${ }^{6}$

\begin{tabular}{|c|c|c|c|c|c|c|c|c|c|c|c|c|c|c|c|c|c|c|}
\hline & \multicolumn{18}{|c|}{ hTAS2R } \\
\hline & 43 & 44 & 46 & 47 & 49 & 13 & 14 & 7 & 9 & 8 & 10 & 1 & 38 & 39 & 40 & 4 & 16 & 41 \\
\hline 1. Quinine & + & + & + & & & & + & + & & & + & & & + & + & + & & \\
\hline 2. Acetaminophen & & & & & & & & & & & & & & + & & & & \\
\hline 3. Azathioprine & & & + & & & & & & & & + & & & & & + & & \\
\hline 4. Caffeine & + & & + & & & & + & + & & & + & & & & & & & \\
\hline 5. Chloramphenicol & + & & + & & & & & & & + & + & + & & + & & & & + \\
\hline 6. Chlorpheniramine & & & + & & & & + & + & & & + & & + & + & + & + & & \\
\hline 7. Colchicine & & & + & & & & & & & & & & & + & & + & & \\
\hline 8. Cromolyn & + & & & & + & & & + & & & & & & & & & & \\
\hline 9. Dextromethorphan & & & & & & & & & & & + & + & & & & & & \\
\hline 10. Diphenhydramine & & & & & & & + & & & & & & & & & & & \\
\hline 11. Diphenidol & + & + & + & + & + & + & + & + & & & + & + & + & + & + & + & + & \\
\hline 12. Erythromycin & & & & & & & & & & & + & & & & & & & \\
\hline 13. Famotidine & & + & & & & & & & & & + & & & & & & & \\
\hline 14. Flufenamic acid & & & & & & & + & & & & & & & & & & & \\
\hline 15. Haloperidol & & & & & & & + & & & & + & & & & & & & \\
\hline 16. Hydrocortisone & & & + & & & & & & & & & & & & & & & \\
\hline 17. Methimazole & & & & & & & & & & & & & + & & & & & \\
\hline 18. Noscapine & & & & & & & + & & & & & & & & & & & \\
\hline 19. Ofloxacin & & & & & & & & & + & & & & & & & & & \\
\hline 20. Papaverine & & & & & & & + & + & & & + & & & & & & & \\
\hline 21. Propylthiouracil & & & & & & & & & & & & & + & & & & & \\
\hline 22. Strychnine & & & + & & & & & & & & + & & & & & & & \\
\hline
\end{tabular}

Table 2. Correlation Coefficients between Taste Sensor Responses and hTAS2R Responses

\begin{tabular}{|c|c|c|c|c|c|c|c|c|c|c|c|c|c|c|c|c|c|c|}
\hline & \multicolumn{18}{|c|}{ hTAS2R } \\
\hline & 43 & 44 & 46 & 47 & 49 & 13 & 14 & 7 & 9 & 8 & 10 & 1 & 38 & 39 & 40 & 4 & 16 & 41 \\
\hline $\mathrm{ACO}$ & 0.04 & 0.25 & -0.05 & 0.29 & 0.09 & 0.29 & $0.72^{a)}$ & 0.39 & -0.16 & -0.16 & 0.31 & 0.25 & 0.13 & 0.17 & $0.53^{b)}$ & 0.27 & 0.29 & -0.16 \\
\hline AN0 & 0.10 & $0.58^{b)}$ & -0.17 & 0.32 & 0.12 & 0.32 & 0.42 & 0.24 & -0.15 & -0.15 & 0.43 & 0.30 & -0.07 & 0.02 & 0.30 & 0.10 & 0.32 & -0.15 \\
\hline BT0 & -0.11 & 0.13 & -0.09 & 0.22 & 0.00 & 0.22 & $0.46^{b)}$ & 0.20 & 0.22 & -0.22 & 0.37 & 0.13 & 0.00 & 0.00 & 0.40 & 0.11 & 0.22 & -0.22 \\
\hline $\mathrm{C} 00$ & -0.09 & 0.05 & -0.09 & -0.13 & -0.19 & -0.13 & -0.30 & -0.38 & 0.36 & 0.36 & -0.06 & 0.05 & -0.29 & 0.08 & -0.24 & -0.09 & -0.13 & 0.36 \\
\hline AE1 & 0.31 & -0.19 & 0.33 & -0.10 & 0.26 & -0.10 & -0.15 & 0.24 & -0.10 & 0.46 & 0.19 & 0.16 & 0.08 & 0.24 & 0.16 & 0.03 & -0.10 & 0.46 \\
\hline
\end{tabular}

a) $p<0.01, b) p<0.05$, (Fisher's exact test). 
not respond to 14 compounds (azathioprine, acetaminophen, erythromycin, ofloxacin, caffeine, cromolyn, chloramphenicol, colchicine, strychnine, hydrocortisone, famotidine, flufenamic acid, propylthiouracil and methimazole) out of the 19 compounds which are not ligands of hTAS2R40. AC0 and hTAS2R40 responses were significantly correlated $(* p<0.05$, Fisher's exact test).

Correlation between responses of AN0 and hTAS2R14: AN0 responded to five compounds (quinine, diphenidol, diphenhydramine, papaverine and haloperidol) out of the nine compounds known to be ligands of hTAS2R14. AN0 did not respond to 11 compounds (azathioprine, acetaminophen, erythromycin, ofloxacin, cromolyn, chloramphenicol, colchicine, strychnine, hydrocortisone, propylthiouracil and methimazol) out of the 13 compounds which are not ligands of hTAS2R14. Correlation between responses of AN0 and hTAS2R14 were close to significance ( $p=0.064$, Fisher's exact test).

Correlation between responses of AN0 and hTAS2R10: AN0 responded to six compounds (quinine, diphenidol, dextromethorphan, papaverine, haloperidol and famotidine) out of the 12 compounds known to be ligands of hTAS2R10; AN0 did not respond to nine compounds (acetaminophen, ofloxacin, cromolyn, colchicine, noscapine, hydrocortisone, fulfenamic acid, propylthiouracil and methimazole) out of the 10 compounds which are not ligands of hTAS2R10. Correlation between responses of AN0 and hTAS2R10 were close to significance ( $p=0.059$, Fisher's exact test).

Correlation between responses of AN0 and hTAS2R44: ANO responded to three compounds (quinine, diphenidol and famotidine) all known to be ligands of hTAS2R44; AN0 did not respond to 15 compounds (azathioprine, acetaminophen, erythromycin, ofloxacin, caffeine, cromolyn, chloramphenicol, chlorpheniramine, colchicine, strychnine, noscapine, hydrocortisone, flufenamic acid, propylthiouracil and methimazole) out of the 19 compounds which are not ligands of hTAS2R44. AN0 and hTAS2R44 responses were significantly correlated (*p<0.05, Fisher's exact test).

Correlation between responses of BT0 and hTAS2R14: BT0 responded to seven compounds (quinine, chlorpheniramine, diphenidol, diphenhydramine, noscapine, papaverine and haloperidol) out of the nine compounds known to be ligands of hTAS2R14; BT0 did not respond to nine compounds (azathioprine, acetaminophen, cromolyn, chloramphenicol, colchicine, hydrocortisone, famotidine, propylthiouracil and methimazole) out of the 13 compounds which are not ligands of hTAS2R14. BT0 and hTAS2R14 responses were significantly correlated $(* p<0.05$, Fisher's exact test).

Correlation between responses of AE1 and hTAS2R8 or hTAS2R41: the correlation coefficients of the responses of AE1 and hTAS2R8 and hTAS2R41 were both 0.46; there was no significant correlation between them by Fisher's exact test.

Correlation between Physicochemical Parameters of the 22 Compounds and the Responses of Taste Sensors and hTAS2Rs The physicochemical parameters of the 22 compounds extracted from the Bitter DB are shown in Table 3. The MW values of erythromycin, ofloxacin, cromolyn, chloramphenicol, colchicine, strychnine, noscapine, papaverine, haloperidol, hydrocortisone and famotidine are larger than that of quinine hydrochloride (MW 324). The $\log P$ values of chlorpheniramine, diphenidol, diphenhydramine, dextromethorphan, noscapine, papaverine, haloperidol and flufenamic acid are greater than that of quinine hydrochloride ( $\log P$ 2.73). The $\log S$ values of azathioprine, acetaminophen, ofloxacin, caffeine, strychnine, noscapine, hydrocortisone, famotidine, propylthiouracil and methimazole are greater than that of quinine hydrochloride $(\log S-4.1)$. The HBD counts of chlorpheniramine, diphenhydramine, strychnine, dextromethorphan, noscapine and papaverine are zero while others are $\geq 1$; quinine is 1 . HBA counts of azathioprine, erythromycin,

Table 3. Physicochemical Parameters of the 22 Compounds Extracted from the Bitter DB ${ }^{6}$

\begin{tabular}{|c|c|c|c|c|c|c|c|}
\hline & MW & $\log P$ & $\log S$ & HBD & HBA & Rings & Aromatic rings \\
\hline Quinine & 324 & 2.73 & -4.14 & 1 & 4 & 4 & 2 \\
\hline Acetaminophen & 151 & 0.71 & -1.39 & 2 & 2 & 1 & 1 \\
\hline Azathioprine & 277 & 1.07 & -2.66 & 1 & 7 & 3 & 3 \\
\hline Caffeine & 194 & -0.10 & -0.64 & 3 & 0 & 2 & 1 \\
\hline Chloramphenicol & 323 & 1.03 & -4.12 & 3 & 5 & 1 & 1 \\
\hline Chlorpheniramine & 275 & 3.70 & -4.97 & 0 & 2 & 2 & 2 \\
\hline Colchicine & 399 & 2.04 & -4.70 & 1 & 6 & 3 & 1 \\
\hline Cromolyn & 468 & 1.77 & -4.33 & 3 & 11 & 4 & 2 \\
\hline Dextromethorphan & 271 & 3.67 & -4.53 & 0 & 2 & 4 & 1 \\
\hline Diphenhydramine & 255 & 3.38 & -4.24 & 0 & 2 & 2 & 2 \\
\hline Diphenidol & 309 & 4.39 & -5.61 & 1 & 2 & 3 & 2 \\
\hline Erythromycin & 734 & 1.79 & -4.76 & 5 & 14 & 3 & 0 \\
\hline Famotidine & 337 & -2.62 & -3.43 & 4 & 9 & 1 & 1 \\
\hline Flufenamic acid & 281 & 3.95 & -4.79 & 2 & 3 & 2 & 2 \\
\hline Haloperidol & 375 & 3.89 & -5.70 & 1 & 3 & 3 & 2 \\
\hline Hydrocortisone & 362 & 1.28 & -3.07 & 3 & 5 & 4 & 0 \\
\hline Methimazole & 114 & 0.41 & -0.92 & 1 & 1 & 1 & 0 \\
\hline Noscapine & 413 & 3.01 & -3.25 & 0 & 8 & 5 & 2 \\
\hline Ofloxacin & 361 & -1.37 & -1.61 & 7 & 1 & 4 & 1 \\
\hline Papaverine & 339 & 3.50 & -5.75 & 0 & 5 & 3 & 3 \\
\hline Propylthiouracil & 170 & 1.58 & -2.55 & 2 & 2 & 1 & 0 \\
\hline Strychnine & 344 & 1.15 & -1.70 & 0 & 3 & 7 & 1 \\
\hline
\end{tabular}


cromolyn, chloramphenicol, colchicine, noscapine, papaverine, hydrocortisone and famotidine are greater than that of quinine hydrochloride, which is 4 . Ring counts of ofloxacin, cromolyn, strychnine, dextromethorphan, noscapine and hydrocortisone are greater than that of quinine hydrochloride, which is 4 . The aromatic ring counts of azathioprine, cromolyn, chlorpheniramine, diphenidol, diphenhydramine, noscapine, papaverine, haloperidol and flufenamic acid are greater than that of quinine hydrochloride, which is 2 .

Correlation coefficients between the physicochemical parameters of the 22 compounds and the taste sensor responses are shown in Table 4 . High $\log P$ values $(\geqq 2.73)$ and the responses of $\mathrm{ACO}, \mathrm{AN} 0$ and $\mathrm{BT} 0$ were significantly correlated $(* * p<0.01$, Fisher's exact test). Correlations between the physicochemical parameters and the responses of hTAS2Rs are shown in Table 5 . High $\log P$ values $(\geqq 2.73)$ and the response of hTAS2R 14 were significantly correlated $(* * p<0.01$, Fisher's exact test).

\section{Discussion}

In the taste map of the 22 compounds used in this study (Fig. 1), haloperidol, diphenidol, diphenhydramine, dextromethorphan and papaverine mapped on the right side (the direction of the $\mathrm{PC} 1$ axis reflects the basic bitterness contribution) of quinine, the standard compound for assessing bitterness. It was therefore predicted that these five compounds would express stronger bitterness than quinine. The outputs of taste sensors AC0, AN0 and BT0 increased in a dose-depen- dent manner in response to these compounds (Fig. 2), with the output values of sensor BT0 being the highest. This suggests that sensor BT0 has the greatest sensitivity for bitterness.

Regarding the correlation between the responses of the taste sensors and those of hTAS2Rs, BT0 was significantly correlated with hTAS2R14 (Table 2). Although AC0 also significantly correlated with hTAS2R14, BT0 was the most sensitive taste sensor for ligands to hTAS2R14.

Taste sensors AC0, AN0 and BT0 reacted more strongly to compounds with high $\log P$ values (Table 4 ). Bitter compounds are adsorbed on the hydrophobic part of the taste sensor membrane and cause a change in membrane potential by changing the charge density, which is reflected by the taste sensor output. ${ }^{12)}$ So high $\log P$ compounds will react more readily with taste sensors. Roland et al. suggested that hydrophobic aromatic features, and therefore hydrophobic interaction with the binding site, is of greater importance in hTAS2R14 than in hTAS2R 39. ${ }^{22)}$ In fact, the $\log P$ values and aromatic ring count of the 22 compounds used in our study correlated well with the responses of hTAS2R14 (Table 5). This supports the finding that taste sensor BT0 would be the most useful taste sensor for evaluating the bitterness of hydrophobic compound which stimulate the bitter taste receptor hTAS2R14.

Recently, it has been revealed that hTAS2R14 recognizes an enormous variety of chemically diverse synthetic and natural bitter compounds, including medicinal drugs. ${ }^{23,24)}$ Inhibition of hTAS2Rs, especially hTAS2R14, would be one approach to reducing the bitterness of drugs. To date, only a

Table 4. Correlation Coefficients between Physicochemical Parameters and the Responses of Taste Sensors to the 22 Compounds

\begin{tabular}{rrrrrrrr}
\hline \hline & MW & $\log P$ & $\log S$ & HBD & HBA & Rings & Aromatic rings \\
\hline AC0 & 0.00 & $0.91^{a)}$ & -0.45 & -0.60 & -0.12 & 0.09 & 0.64 \\
AN0 & 0.10 & $0.62^{a)}$ & -0.36 & -0.24 & -0.04 & -0.05 & 0.36 \\
BT0 & 0.27 & $0.65^{a)}$ & -0.37 & -0.61 & -0.18 & 0.29 & 0.37 \\
C00 & -0.20 & -0.30 & 0.35 & 0.38 & 0.06 & -0.20 & -0.15 \\
AE1 & 0.00 & -0.15 & -0.04 & -0.24 & 0.04 & 0.18 & 0.04 \\
\hline
\end{tabular}

a) $p<0.01$ (Fisher's exact test).

Table 5. Correlation Coefficients between Physicochemical Parameters and the Responses of hTAS2Rs to the 22 Compounds

\begin{tabular}{|c|c|c|c|c|c|c|c|}
\hline & MW & $\log P$ & $\log S$ & HBD & HBA & Rings & Aromatic rings \\
\hline R43 & -0.11 & -0.01 & 0.06 & 0.33 & 0.16 & 0.10 & 0.16 \\
\hline R44 & 0.13 & 0.21 & 0.10 & 0.24 & 0.17 & 0.01 & 0.17 \\
\hline R46 & -0.09 & -0.13 & 0.20 & 0.09 & 0.17 & 0.03 & -0.02 \\
\hline R47 & -0.22 & 0.26 & -0.24 & 0.13 & -0.20 & -0.15 & 0.24 \\
\hline R49 & 0.00 & 0.06 & -0.35 & 0.19 & 0.03 & 0.12 & 0.35 \\
\hline $\mathrm{R} 13$ & -0.22 & 0.26 & -0.24 & 0.13 & -0.20 & -0.15 & 0.24 \\
\hline $\mathrm{R} 14$ & -0.09 & $0.81^{a)}$ & -0.35 & -0.32 & -0.20 & -0.17 & $0.73^{a)}$ \\
\hline R7 & 0.00 & 0.32 & -0.26 & -0.08 & 0.06 & 0.02 & 0.47 \\
\hline R9 & 0.22 & -0.18 & 0.20 & 0.13 & -0.20 & 0.32 & -0.20 \\
\hline $\mathrm{R} 8$ & -0.22 & -0.18 & 0.20 & 0.13 & 0.24 & -0.15 & -0.20 \\
\hline $\mathrm{R} 10$ & 0.00 & 0.20 & -0.10 & -0.15 & 0.10 & -0.16 & 0.10 \\
\hline $\mathrm{R} 1$ & -0.40 & 0.21 & -0.17 & -0.05 & -0.10 & 0.01 & -0.10 \\
\hline R38 & -0.47 & 0.09 & -0.04 & 0.02 & -0.43 & -0.32 & 0.04 \\
\hline R39 & -0.20 & 0.11 & -0.06 & 0.15 & 0.06 & -0.20 & 0.06 \\
\hline R40 & -0.13 & 0.48 & -0.17 & -0.05 & -0.10 & 0.01 & 0.44 \\
\hline R4 & -0.11 & 0.21 & -0.16 & 0.09 & 0.16 & -0.14 & 0.38 \\
\hline $\mathrm{R} 16$ & -0.22 & 0.26 & -0.24 & 0.13 & -0.20 & -0.15 & 0.24 \\
\hline R41 & -0.22 & -0.18 & 0.20 & 0.13 & 0.24 & -0.15 & -0.20 \\
\hline
\end{tabular}

a) $p<0.01$ (Fisher's exact test). 
few bitter inhibitors have been discovered that block bittersubstance-evoked hTAS2R activation, such as Sakuranetin for hTAS2R31, ${ }^{25,26)}$ 6-methoxyflavanones for hTAS2R39, ${ }^{27,28)}$ $\gamma$-aminobutyric acid (GABA), $\quad N^{\alpha}, N^{\alpha}$-bis(carboxymethyl)L-lysine $(\mathrm{BCML})^{29)}$ and abscisic $\operatorname{acid}^{30)}$ for hTAS2R4, and (R)-Citronellal for hTAS2R43 and hTAS2R46. ${ }^{31)}$ Batenburg et al. identified several hTAS2R14 receptor antagonists from a screening of synthetic compounds; however, the nature of these compounds did not allow their effects to be validated in vivo before extensive safety evaluation. ${ }^{32)}$ Taste sensor BT0, which has high sensitivity and whose responses correlated well with the responses of hTAS2R14, should therefore be useful for screening potential inhibitors of bitter taste receptor hTAS2R14.

Although not only hTAS2R14 but also hTAS2R10 are broadly tuned by a large number of agonists, ${ }^{3,4,33)}$ there was no significant correlation between the responses of hTAS2R10 and $\log P \geqq 2.73$. It seems that the responses of hTAS2R10 were unrelated to the hydrophobicity of the ligand, unlike those of hTAS2R14. Born et al. demonstrated, using molecular modelling, point mutagenesis and functional assays, that the binding pocket of hTAS2R10 is not optimized for high-affinity interactions with a handful of ligands but is rather designed to accommodate many diverse agonists. ${ }^{33)}$ In fact, the ligands of hTAS2R10 have diverse $\log P$ values.

The six compounds which were predicted to express the strongest bitterness in our study (Figs. 1, 2) were all ligands of hTAS2R14 and/or hTAS2R10, both of them were broadly tuned by a large number of agonists. Our findings of a correlation between the responses of hTAS2R 14 and 10 and those of BT0, AC0, and AN0 suggest that a large number of bitter compounds, as ligands of the bitter taste receptor, could be detected by these taste sensors.

\section{Conclusion}

Taste sensor BT0 had high sensitivity compared to AC0 or AN0 and the responses of BT0 and those of hTAS2R14 was significantly correlated. High $\log P$ values and response to hTAS2R14 (and BT0) were also significantly correlated. These results suggest that taste sensor BT0, which has high sensitivity as well as a significant correlation with response to hTAS2R14, would be useful in evaluating the bitterness of hydrophobic compounds which respond to hTAS2R14 and also in screening potential bitterness inhibitors.

There are many reports on evaluation of the bitterness of drugs using taste sensors, however, this is the first report evaluating the correlation between the response of the taste sensor and the response of human bitter taste receptors.

Acknowledgments This work was supported by the Japan Society for the Promotion of Science (JSPS) KAKENHI Grant Numbers JP16K08426, JP16K18970.

Conflict of Interest The authors declare no conflict of interest.

\section{References}

1) Jaggupilli A., Howard R., Upadhyaya J. D., Bhullar R. P., Chelikani P., Int. J. Biochem. Cell Biol., 77 (Pt. B), 184-196 (2016).

2) Adler E., Hoon M. A., Mueller K. L., Chandrashekar J., Ryba N. J., Zuker C. S., Cell, 100, 693-702 (2000).
3) Meyerhof W., Batram C., Kuhn C., Brockhoff A., Chudoba E., Bufe B., Appendino G., Behrens M., Chem. Senses, 35, 157-170 (2010).

4) Behrens M., Meyerhof W., Semin. Cell Dev. Biol., 24, 215-221 (2013).

5) Di Pizio A., Niv M. Y., Bioorg. Med. Chem., 23, 4082-4091 (2015).

6) Wiener A., Shudler M., Levit A., Niv M. Y., Nucleic Acids Res., 40 (D1), D413-D419 (2012).

7) Mistry P., Batchelor H., SPaeDD-UK project (Smart Paediatric Drug Development-U.K.), J. Pharm. Pharmacol., 69, 361-376 (2017).

8) Batchelor H., Venables R., Marriott J., Mills T., Int. J. Pharm., 479, 277-281 (2015)

9) Liu F., Ghaffur A., Bains J., Hamdy S., Int. J. Pharm., 512, 374-381 (2016).

10) Petrovick G. F., Breitkreutz J., Pein-Hackelbusch M., Int. J. Pharm., 506, 361-370 (2016).

11) Immohr L. I., Hedfeld C., Lang A., Pein-Hackelbusch M., AAPS PharmSciTech, 18, 330-340 (2017).

12) Kobayashi Y., Habara M., Ikezaki H., Chen R., Naito Y., Toko K., Sensors, 10, 3411-3443 (2010).

13) Woertz K., Tissen C., Kleinebudde P., Breitkreutz J., J. Pharm. Biomed. Anal., 55, 272-281 (2011).

14) Ito M., Yoshida M., Kobayashi Y., Hiraoka M., Ikezaki H., Uchida T., Sensors and Materials, 23, 483-492 (2011).

15) Uchida T., Yoshida M., Hazekawa M., Haraguchi T., Furuno H., Teraoka M., Ikezaki H., J. Pharm. Pharmacol., 65, 1312-1320 (2013).

16) Yoshida M., Haraguchi T., Uchida T., Chem. Pharm. Bull., 62, 1252-1258 (2014).

17) Haraguchi T., Miyazaki A., Yoshida M., Uchida T., J. Pharm. Pharmacol., 65, 980-987 (2013).

18) Maniruzzaman M., Douroumis D., J. Pharm. Pharmacol., 67, 43-55 (2015).

19) Nakamura H., Uchida S., Sugiura T., Namiki N., Int. J. Pharm., 493, 305-312 (2015).

20) Ghose A. K., Crippen G. M., J. Comput. Chem., 7, 565-577 (1986).

21) Tetko I. V., Tanchuk V. Y., Kasheva T. N., Villa A. E., J. Chem. Inf. Comput. Sci., 41, 1488-1493 (2001).

22) Roland W. S., van Buren L., Gruppen H., Driesse M., Gouka R. J., Smit G., Vincken J. P., J. Agric. Food Chem., 61, 10454-10466 (2013).

23) Levit A., Nowak S., Peters M., Wiener A., Meyerhof W., Behrens M., Niv M. Y., FASEB J., 28, 1181-1197 (2014).

24) Karaman R., Nowak S., Di Pizio A., Kitaneh H., Abu-Jaish A., Meyerhof W., Niv M. Y., Behren M., Chem. Biol. Drug Des., 88, 66-75 (2016)

25) Slack J. P., Brockhoff A., Batram C., Menzel S., Sonnabend C., Born S., Galindo M. M., Kohl S., Thalmann S., Ostopovici-Halip L., Simons C. T., Ungureanu I., Duineveld K., Bologa C. G., Behrens M., Furrer S., Oprea T. I., Meyerhof W., Curr. Biol., 20, 1104-1109 (2010).

26) Fletcher J. N., Kinghorn A. D., Slack J. P., McCluskey T. S., Odley A., Jia Z., J. Agric. Food Chem., 59, 13117-13121 (2011).

27) Roland W. S., Gouka R. J., Gruppen H., Driesse M., van Buren L., Smit G., Vincken J. P., PLOS ONE, 9, e94451 (2014).

28) Roland W. S., Sanders M. P., van Buren L., Gouka R. J., Gruppen H., Vincken J. P., Ritschel T., PLOS ONE, 10, e0118200 (2015).

29) Pydi S. P., Sobotkiewicz T., Billakanti R., Bhullar R. P., Loewen M. C., Chelikani P., J. Biol. Chem., 289, 25054-25066 (2014).

30) Pydi S. P., Jaggupilli A., Nelson K. M., Abrams S. R., Bhullar R. P., Loewen M. C., Chelikani P., Biochemistry, 54, 2622-2631 (2015).

31) Suess B., Brockhoff A., Meyerhof W., Hofmann T., J. Agric. Food Chem., in press.

32) Batenburg A. M., de Joode T., Gouka R. J., J. Agric. Food Chem., 64, 2619-2626 (2016).

33) Born S., Levit A., Niv M. Y., Meyerhof W., Behrens M., J. Neurosci., 33, 201-213 (2013). 